Check for updates

Cite this: RSC Adv., 2018, 8, 10620

Received 14th January 2018

Accepted 1st March 2018

DOI: $10.1039 / \mathrm{c} 8 \mathrm{ra00401c}$

rsc.li/rsc-advances

\section{Biodegradable copolypeptide hydrogel prodrug accelerates dermal wound regeneration by enhanced angiogenesis and epithelialization}

\author{
Anqi Chen, ${ }^{\text {abc }}$ Huacheng $\mathrm{He}$, (D) *c Guanglong Ma, ${ }^{\mathrm{d}} \mathrm{Yi} \mathrm{Li}^{\mathrm{b}}{ }^{\mathrm{b}}$ Shishuang Jiang, ${ }^{\mathrm{b}}$

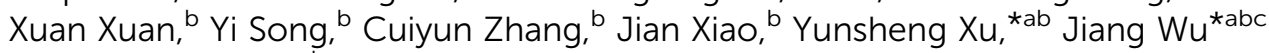 \\ and Shengfu Chen ${ }^{\text {d }}$
}

Hydrogels are one of the most promising wound dressings. However, their effectiveness on wound healing is still largely limited due to either the non-degradability or the release of non-therapeutic degradable products. Herein, a biodegradable copolypeptide hydrogel based on the glutamic acid and lysine was synthesized and applied as both wound dressing and therapeutic prodrug. The hydrogel can degrade in the existence of elevated degradative enzymes in a wound environment, which will release therapeutic amino acids to enhance the wound healing. In vivo results found that the hydrogel could effectively promote wound regeneration in both macroscopic and microscopic scales. Further investigation revealed that the wound healing effect of the hydrogel was highly attributed to its enhanced impact on angiogenesis, cell proliferation and re-epithelialization of the wound. All in all, the present study proves that the degradable copolypeptide hydrogel can efficiently improve wound healing and indicates its potent clinical application for wound regeneration.

\section{Introduction}

Dermal trauma is one of the commonest clinical issues, while the satisfying regeneration and outcome of this issue remains a big challenge due to its complicated overlapping healing processes and obscure underlying mechanisms. Moreover, although the human skin can achieve self-regeneration itself after injury, this capacity may be compromised under specific conditions such as diabetes, which makes wound healing a more serious global clinical issue and burden to healthcare systems worldwide. ${ }^{1}$ To overcome this clinical problem, a lot of efforts have been made, among which the development of wound dressings has especially broad prospects. However, despite of the recent considerable advances in wound dressings, only a few of them were approved for clinical application and an ideal dressing that can fulfil the desirable requisites still needs further investigation. ${ }^{2}$

Among various wound dressings, hydrogels are one of the most promising ones. Hydrogel can maintain the wound with a moist micro-environment, which was believed to favor the migration of keratinocytes thus promoting re-epithelialization

${ }^{a}$ The First Affiliated Hospital of Wenzhou Medical University, Wenzhou, 325000, P. R. China. E-mail: xuyunsheng1018@163.com

${ }^{b}$ Wenzhou Medical University, Wenzhou, 325035, P. R. China.E-mail:woody870402@ hotmail.com

'Wenzhou University, Wenzhou, 325035, P. R. China.E-mail: hehc@wzu.edu.cn

${ }^{d}$ Zhejiang University, Hangzhou, 310027, P. R. China process in the wound healing process. Moreover, hydrogel dressings can absorb wound exudates and prevent external bacterial contamination, ${ }^{3}$ both of which will greatly benefit the wound regeneration. However, due to the non-degradability and the tight adhesive to the regenerated skin, the removal of the hydrogel often causes secondary injury to the skin, thus deteriorating its therapeutic effect. Therefore, degradable hydrogel based wound dressings are attracting increasing interest. This type of hydrogels usually consists of highly biocompatible macromolecules, including polysaccharides, collagens, polymers and peptides. ${ }^{4-6}$ These macromolecules generally degrade to non-toxic small molecules to avoid the further removal of hydrogels after wound regeneration. Typically, the degradative small molecule products are non-therapeutic, the impact of the degradable hydrogel on wound regeneration are highly relied on the hydrogel itself, which is very similar to those nondegradable ones. To further improve the wound healing efficacy, therapeutic agents are often encapsulated into the hydrogels. In these cases, the hydrogels not only provide as wound dressings, but also serve as carriers to release therapeutic drugs to reach enhanced would regeneration. Nevertheless, this kind of hydrogels is much more complicated, and the loading and release of the encapsulated drugs may be extremely hard to control. Thus, to design a novel hydrogel which functions as wound dressing and therapeutic prodrug simultaneously will be more promising.

Recently, we designed a degradable hydrogel based on the glutamic acid and lysine peptides. ${ }^{7}$ The hydrogel was proved to 
be degraded in the high level of degradative enzyme environment such as tumor and inflammation sites. Importantly, the degradative small molecules include glutamic acid and lysine, both of which have been reported to benefit the wound healing. The treatment of wounds with glutamic acid has been proved to enhance angiogenesis and collagen deposition, while the deficiency of lysine in wound has been reported to lead to the failure of collagen deposition in the wound, ${ }^{8,9}$ and thus results in detrimental wound repair. Obviously, our degradable polypeptide hydrogel can serve as both wound dressing and therapeutic prodrug, making it an ideal system for the wound therapy.

Therefore, in this study, a biodegradable copolypeptide hydrogel was synthesized and applied as wound dressing and prodrug to treat dermal wound. The hydrogel composed of $\mathrm{L}$ glutamic acid and L-lysine copolypeptide, which could be degraded in the high degradative enzyme environment in the wound healing process to release free amino acids. The hydrogel together with the released amino acids took effect on the wound healing. In vivo study found that the hydrogel could effectively promote wound regeneration in both macroscopic and microscopic scales. The mechanism of the hydrogel on wound healing was extensively investigated and it was found that the hydrogel improved wound recovery by boosting angiogenesis, increasing cell proliferation and accelerating reepithelialization.

\section{Materials and methods}

\subsection{Materials and reagents}

The $N \varepsilon$-carbobenzyloxy-L-lysine $(\operatorname{Lys}(\mathrm{Z}))$ and $\gamma$-benzyl-L-glutamate (Glu(OBZL)) were obtained from GL Biochem Ltd, Shanghai, China. 1-Ethyl-3-(3-dimethylaminopropyl), carbodiimide hydrochloride (EDC $\cdot \mathrm{HCl}$ ), trifluoroacetic acid (TFA), $n$ hexylamine were purchased from Aladdin Reagent Co., Ltd, Shanghai, China. Anhydrous dimethylformamide (DMF) was purchased from Alfa Aesar, MA, USA. $33 \mathrm{wt} \% \mathrm{HBr} / \mathrm{HOAc}$ solution was obtained from Sigma-Aldrich Co. LLC, MO, USA.

\subsection{Methods}

2.2.1 Poly(L-glutamic acid-co-L-lysine) copolypeptide synthesis. $N$-Carboxyanhydride (NCA) of Glu(OBZL) and NCA of Lys(Z) were synthesized as previously described. ${ }^{10,11}$ The poly(Lglutamic acid-co-L-lysine) copolypeptide was synthesized through the copolymerization of NCA of Glu(OBZL) and NCA of Lys(Z) with the initiator of $n$-hexylamine. In brief, $0.81 \mathrm{~g}$ NCA of Glu(OBZL) (3.06 mmol) and $0.918 \mathrm{~g}$ NCA of Lys(Z) (3 mmol) were dissolved in $10 \mathrm{~mL}$ anhydrous DMF in nitrogen atmosphere followed by the addition of $0.003 \mathrm{~g} n$-hexylamine $(0.03 \mathrm{mmol})$ for $48 \mathrm{~h}$ at room temperature. The solution was then precipitated in diethyl ether thrice. After filtration, the precipitate was dried in vacuum overnight to obtain a white solid, which was then dissolved in $10 \mathrm{~mL}$ TFA in ice water environment, followed by the addition of $10 \mathrm{~mL} 33 \mathrm{wt} \% \mathrm{HBr} / \mathrm{HOAC}$. After $4 \mathrm{~h}$ agitation, the mixture was precipitated into excess ethyl ether, and then washed with ethyl ether twice. The precipitate was then dried in vacuum oven for $24 \mathrm{~h}$, followed by the dialysis against phosphate buffer saline (PBS) (pH 7.4) and distilled water each for one day. The dialysis buffer was changed every 6 h. Finally, the copolypeptide was obtained after freeze-drying. The structure of the polypeptide was verified by ${ }^{1} \mathrm{H}$ nuclear magnetic resonance using a Bruker ADVANCE2B/400 MHz apparatus. The molecular weight and polydispersity of the copolypeptide was measured by gel permeation chromatography (GPC) equipped with refractive index detector (Shimadzu).

2.2.2 Hydrogels preparation. After the dissolution of the copolypeptide in PBS (pH 6.5, $0.1 \mathrm{~g} \mathrm{~mL} \mathrm{~m}^{-1}$ ), $\mathrm{EDC} \cdot \mathrm{HCl}$ was added as a coupling reagent at different molar ratios of $10 \%, 20 \%, 30 \%$ to carboxyl group or amine group, respectively. After vortex for 30 seconds, the mixture was poured into a cylindroid vessel and the hydrogels were formed within $5 \mathrm{~min}$. The hydrogels were then placed at $37^{\circ} \mathrm{C}$ for $4 \mathrm{~h}$ to achieve complete reaction, and in PBS (pH 6.5) for $24 \mathrm{~h}$ and in PBS (pH 7.4) for another $48 \mathrm{~h}$ to remove the unreacted copolypeptide and the EDC $\cdot \mathrm{HCl}$. The morphology of the hydrogels was characterized by scanning electron microscope (SEM, S1510, Hitachi).

2.2.3 Equilibrium swelling of hydrogels. The hydrogels were immersed in $\mathrm{PBS}(\mathrm{pH} 7.4)$ at $37^{\circ} \mathrm{C}$ for $48 \mathrm{~h}$ to investigate their water absorptivity. The swollen hydrogels were weighed $\left(W_{\text {swollen }}\right)$, and then the hydrogels were lyophilized and weighed $\left(W_{\mathrm{dry}}\right)$ again. The swelling ratio of the hydrogels were defined by the following equation:

$$
\text { Swelling ratio }=\left(W_{\text {swollen }}-W_{\text {dry }}\right) / W_{\text {dry }}
$$

2.2.4 Animal model for dermal wound healing. Male C57BL/6 mice (8-10 weeks) were obtained from the Laboratory Animals Center of Wenzhou Medical University. The animal experimental procedures were accomplished in the Laboratory Animals Center of Wenzhou Medical University and approved by the Ethics Committee of Wenzhou Medical University (approval no. Wydw2017-0501). International ethical guidelines and the National Institutes of Health Guide concerning the Care and Use of Laboratory Animals were strictly followed. Mice were anesthetized with $4 \%$ chloral hydrate and the skin on the back was shaved with a depilator and depilatory cream, and sterilized with $70 \%$ ethanol. Silicone rings with an internal diameter of $8 \mathrm{~mm}$ and thickness of $0.5 \mathrm{~mm}$ were stitched on the skin. A $6 \mathrm{~mm}$ biopsy punch (Acuderm ${ }^{\circledR}$ inc., Ft Lauderdale, FL, USA) was used to make two full-thickness wounds per mice on their central back. Photographs were taken of each wound. Mice were randomly divided into two groups and were treated with $0.9 \%$ saline and hydrogels, respectively. Hydrogels with a diameter of $7 \mathrm{~mm}$ was applied directly to each wound, after which the wound was covered with a sheet of 3M Tegaderm Film (3M Health Care, Germany) and medical bandages. Photographs of the wounds were taken on day 7, 10, 14, 17, 20 post wounding and analyzed using Image-Pro plus. On day 7 and 20 after surgery, mice were sacrificed after anaesthesia and wounds were excised and fixed in $4 \%$ paraformaldehyde at $4{ }^{\circ} \mathrm{C}$ for $24 \mathrm{~h}$. After paraffin embedding, wound tissues were sectioned at a thickness of $5 \mu \mathrm{m}$ using 
a microtome (LEICA RM2235, Germany) and then placed in a constant temperature oven at $65{ }^{\circ} \mathrm{C}$ for $4 \mathrm{~h}$.

2.2.5 Haematoxylin and eosin staining. Sections were deparaffinized in xylene for 30 minutes and rehydrated in ethanol solutions with descending concentrations of $100 \%$, $95 \%$ and $80 \%$, and distilled water for $5 \mathrm{~min}$ respectively. Samples were submerged in haematoxylin (Beyotime Institute of Biotechnology, China) for $5 \mathrm{~min}$, followed by $3 \mathrm{~min}$ in phosphate-buffered saline (PBS) solution to reduce the background staining. Sections were then stained with eosin (Beyotime Institute of Biotechnology, China) for $2 \mathrm{~min}$, followed by $5 \mathrm{~min}$ in distilled water, $5 \mathrm{~min}$ in $80 \%, 90 \%$ and $100 \%$ ethanol respectively for dehydration, and $15 \mathrm{~min}$ in xylene. Slides were mounted with neutral resin and covered by coverslips. The stained sections were observed and imaged using a Nikon microscope (Nikon, Tokyo, Japan).

2.2.6 Masson's trichrome staining. Sections were deparaffinized and rehydrated as mentioned above and then stained using Masson's trichrome staining kit (Beyotime) following the protocols. Briefly, the cell nuclei were stained with A1 : A2 (1:1) for $5 \mathrm{~min}$, and then sections were thoroughly rinsed with water and submerged in acid alcohol for differentiation for 3 s. Ponceau acid fuchsine solution was used to stain fibrous tissue for $5 \mathrm{~min}$, followed by $1 \mathrm{~min}$ in $2 \%$ acetic acid solution, $30 \mathrm{~s}$ in phosphomolybdic acid solution for differentiation, and $20 \mathrm{~s}$ in aniline blue, after which sections were dehydrated, mounted and coverslipped as mentioned above. The stained sections were observed and images were captured using a Nikon microscope (Nikon, Tokyo, Japan).

2.2.7 Immunofluorescence examinations. After deparaffinization and rehydration, the endogenous peroxidase of the sections was inactivated with $3 \%$ hydrogen peroxide at room temperature for $15 \mathrm{~min}$ and the nonspecific binding sites were blocked with $5 \%$ bovine serum albumin (BSA) at $37{ }^{\circ} \mathrm{C}$ for $30 \mathrm{~min}$. For cytokeratin and PCNA double staining, sections were incubated with a rabbit polyclonal antibody to cytokeratin (1: 200, ab9377, Abcam) and a mouse monoclonal antibody to PCNA (sc25280, 1 : 100, Santa Cruz Biotech, CA, USA) diluted in phosphate-buffered saline (PBS) containing $1 \%$ BSA at $4{ }^{\circ} \mathrm{C}$ overnight and washed with PBS, followed by incubation with a donkey anti-rabbit IgG Alexa Fluor® 488-conjugated secondary antibody (1:1000, ab150073, Abcam) and a donkey anti-mouse IgG Alexa Fluor ${ }^{\circledR}$ 647-conjugated secondary antibody (1: 1000, ab150111, Abcam) diluted in PBS at $37{ }^{\circ} \mathrm{C}$ for $60 \mathrm{~min}$. For CD31 and $\alpha$-SMA double staining, sections were incubated with a rabbit polyclonal antibody to CD31 (1:200, ab28364, Abcam) and a mouse polyclonal antibody to $\alpha$-SMA (1 : 200, ab7817, Abcam) diluted in PBS containing 1\% BSA at $4{ }^{\circ} \mathrm{C}$ overnight and washed with PBS, followed by incubation with a donkey anti-mouse IgG Alexa Fluor® 488-conjugated secondary antibody (1:1000, ab150105, Abcam) and a goat antirabbit IgG Alexa Fluor ${ }^{\circledR}$ 647-conjugated secondary antibody (1 : 1000, ab150075, Abcam) diluted in PBS at $37^{\circ} \mathrm{C}$ for $60 \mathrm{~min}$ in the dark. Sections were then counterstained with DAPI (Beyotime) and mounted with antifade mounting medium.
Fluorescent images were taken using a Nikon confocal laser microscope (Nikon, A1 PLUS, Tokyo, Japan).

2.2.8 Statistical analysis. All data were expressed as mean \pm standard deviations (SD). Statistical differences were performed using one-way analysis of variance (ANOVA) followed by Tukey's test with GraphPad Prism 5 software (GraphPad Software Inc., La Jolla, CA, USA). For all tests, ${ }^{*} p$ value $<0.05,{ }^{* *} p$ value $<0.01$, $* * * p$ value $<0.001$

\section{Results and discussion}

\subsection{Preparation and characterization of hydrogels}

We firstly synthesized the copolypeptide poly(L-glutamic acidco-L-lysine) by ring-opening polymerization of Glu(OBZL)-NCA and Lys(Z)-NCA according to the scheme shown in Fig. 1A. As reported in previous research, ${ }^{7}$ the copolypeptide had a $1: 1$

A
(1)

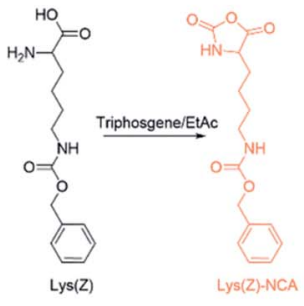

(2)

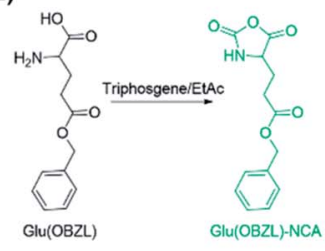

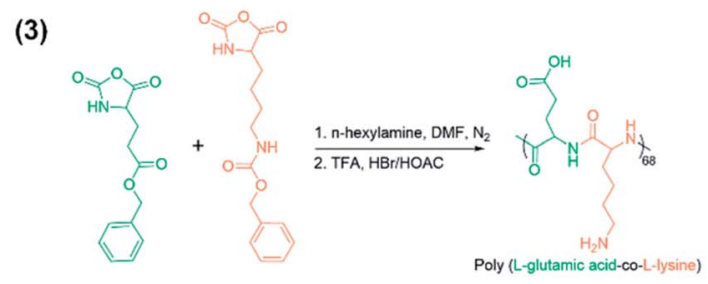

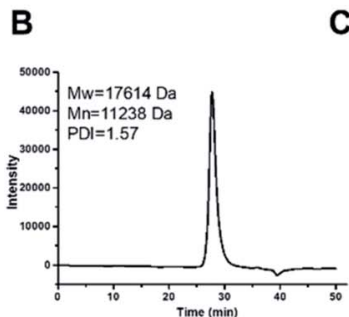

C
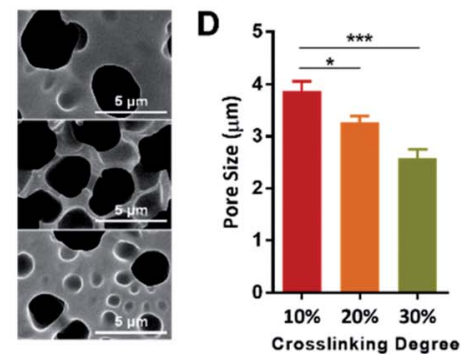

$\mathbf{E}$

\begin{tabular}{|c|c|c|c|}
\hline Degrees of Crosslinking & $10 \%$ & $20 \%$ & $30 \%$ \\
\hline Pore Size $(\mu \mathrm{m})$ & $3.88 \pm 0.67$ & $3.27 \pm 0.40$ & $2.58 \pm 0.57$ \\
\hline Swelling Ratio $(\mathrm{w} / \mathrm{w})$ & $6.69 \pm 0.19$ & $6.06 \pm 0.07$ & $5.84 \pm 0.12$ \\
\hline
\end{tabular}

Fig. 1 Copolypeptide and hydrogels preparation and characterization. (A) Schematic illustration of the synthesis of poly(L-glutamic acid-CoL-lysine) peptide. (B) Representative GPC result indicates that the poly(L-glutamic acid-CO-L-lysine) peptide has a molecular weight around $17.6 \mathrm{kDa}$ with PDI equal to 1.57. (C) SEM images of lyophilized copolypeptide hydrogels with different crosslinking degrees (from top to bottom: $10 \%, 20 \%$ and $30 \%$ ). The scale bars are $5 \mu \mathrm{m}$. (D) Statistics of the pore sizes of the copolypeptide hydrogels with different crosslinking degrees. $* P$ value $<0.05$ and $* * * P$ value $<0.001$. (E) The summary of pore sizes and swelling ratios of copolypeptide hydrogels with different crosslinking degrees. 
ratio between glutamic acid and lysine since the copolypeptide exhibited a well balanced charge property. In addition, GPC result showed that the polypeptide had a molecular weight around $17.6 \mathrm{kDa}$ (68 repeat units) with a polydispersity index (PDI) value equal to 1.57 (Fig. 1B). Due to the high molecular weight $(>10 \mathrm{kDa})$, the peptide could be facilely fabricated into hydrogels. ${ }^{7}$ Therefore, here in our study, three hydrogels with crosslinking degrees of $10 \%, 20 \%$, and $30 \%\left(\mathrm{~mol} \mathrm{~mol}^{-1}\right)$ were firstly prepared according to our previous reports, ${ }^{7}$ by simply adjusting the molar ratio of the coupling reagent, $\mathrm{EDC} \cdot \mathrm{HCl}$, to the total primary amines amount (or carboxyl groups) in the copolypeptide. To be mentioned, herein the crosslinking degree was expressed as the molar percentage of EDC of the total amount of primary amines in the copolypeptide hydrogels. The microstructure of the hydrogels was initially characterized by SEM. As shown in Fig. 1C, the SEM images of the copolypeptide hydrogels indicated that the pore sizes of the hydrogels decreased with the increasing crosslinking density. The hydrogel with $10 \%$ crosslinking extent had an average pore size of $3.88 \pm 0.67 \mu \mathrm{m}$, which was statistically bigger than that of $20 \%$ and $30 \%$ crosslinking extent, whose pore sizes were $3.27 \pm 0.40$ $\mu \mathrm{m}$ and $2.58 \pm 0.57 \mu \mathrm{m}$, respectively (Fig. 1D and E). The big pore size may facilitate water transport and oxygen and nutrients exchange between the external environment and the regenerating cells in the wound site, leading to a better wound healing efficiency. ${ }^{12}$ Since wound exudates absorption by hydrogels may benefit wound healing, ${ }^{13}$ we next investigated the capability of the hydrogels in absorbing fluid by measuring their swelling ratios. After being saturated in $\mathrm{PBS}(\mathrm{pH}=7.4)$, the hydrogels with the degrees of crosslinking of $10 \%, 20 \%$, and $30 \%$ exhibited swelling ratios $(\mathrm{w} / \mathrm{w})$ of $6.69 \pm 0.19,6.06 \pm 0.07$, and $5.84 \pm 0.12$, respectively (Fig. 1E). It revealed that increasing the crosslinking degree reduced swelling ratios of the hydrogels, which was consistent with previous reports. ${ }^{\mathbf{1 4 1 5}}$ This could be explained that with the increase of the crosslinking degrees, there would be more coupling between amine groups and carboxyl groups, which resulted in more covalent bonds among the copolypeptide chains and finally made the hydrogels hard to swell.

\subsection{The hydrogel promotes wound regeneration in both macroscopic and microscopic scales}

Since the $10 \%$ crosslinked hydrogel shows better properties such as bigger average pore size and higher swelling ratio, as well as lower protein absorption with effective enzymatic degradation behavior, ${ }^{7}$ it was chosen to further investigate the in vivo healing-promoting function. The hydrogel was placed directly onto a full-thickness wound healing model of mice, and the wound healing process was carefully monitored. Fig. 2A and B showed the macroscopic images of the wounds on day 0 , day 7 , day 10 , day 14 , day 17 , day 20 post wounding, respectively. It was intuitive and obvious that, compared to the control group which received $0.9 \%$ saline, the wounds treated with the hydrogel had accelerated healing rate during the 20 day process. Based on the wound images, the wound healing closure rates which represented by the percentage of the wound area size out
A

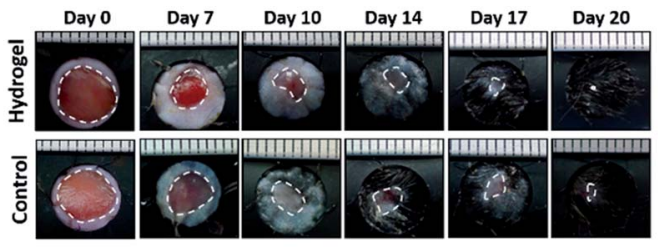

B
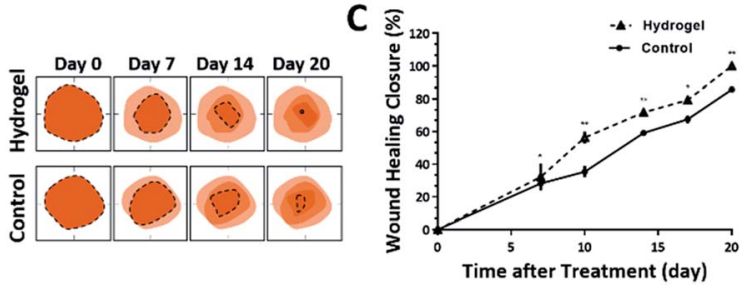

Fig. 2 Copolypeptide hydrogel accelerates wound healing. (A) Representative images of wounds of the $10 \%$ copolypeptide hydrogel group and the control group over the course of healing process. The unit of the rulers over the pictures represents 1 millimeter. (B) Schematic diagram of the wound closure process of the two groups. (C) Wound healing closure rates of these two groups. The rates were presented as a percentage of the initial wound area on day 0 . Statistical differences were performed using one-way analysis of variance (ANOVA). ${ }^{* *} P<0.01, * P<0.05$, compared to control group, $n>6$.

of the initial wound size on day 0 were also summarized in Fig. 2C. The quantitative result of the wound size confirmed that the hydrogel apparently increased the wound closure from day 7 compared to the control, which were $32.14 \pm 8.29 \%$ vs. $28.14 \pm 3.54 \%$ on day $7,56.40 \pm 3.62 \%$ vs. $35.31 \pm 3.5 \%$ on day $10,71.75 \pm 1.73 \%$ vs. $59.16 \pm 1.08 \%$ on day $14,79.35 \pm 2.22 \%$ vs. $67.53 \pm 2.43 \%$ on day 17 and $100.00 \%$ vs. $85.74 \pm 1.60 \%$ on day 20. All the above data verified that the copolypeptide hydrogel could effectively promote wound regeneration in the macroscopic scale in vivo.

Although the hydrogel could improve the wound closure microscopically, its microscopical impact on the wound, in terms of granulation tissue maturation, collagen deposition and skin accessories regeneration (Fig. 3A), is an even more important indicator for the evaluation of the effectiveness of the hydrogel on the recovery of the injured skin. ${ }^{\mathbf{1 6 - 1 8}}$ Therefore, haematoxylin and eosin (H\&E) staining and Masson's trichrome (MT) staining were applied to scrutinize the microscopic wound change after the treatment of the hydrogel. The new epidermal and granulation tissue freshly obtained from the wound sites on day 20 were sectioned and observed with microscope. As indicated by the black arrowheads in Fig. 3B-a and b, the wound treated with the hydrogel had narrower granulation tissue gap compared with the control group, and the gap difference of the two groups was around $0.91 \pm 0.22 \mathrm{~mm}$ (Fig. 3D). Furthermore, based on the H\&E result in Fig. 3B, additional statistical analysis exhibited that the hydrogel treated wounds formed thicker granulation tissue which was $71.21 \pm 3.55 \%$ compared with $42.56 \pm 8.66 \%$ of the control (Fig. 3E). All these data proving that the hydrogel could induce wound regeneration in a more normal way. The collagen deposition of the wound was also investigated by MT staining to further evaluate the normalization degree of collagen in the injured skin. As shown in Fig. 3C, the hydrogel treated wound exhibited much darker and even 
A

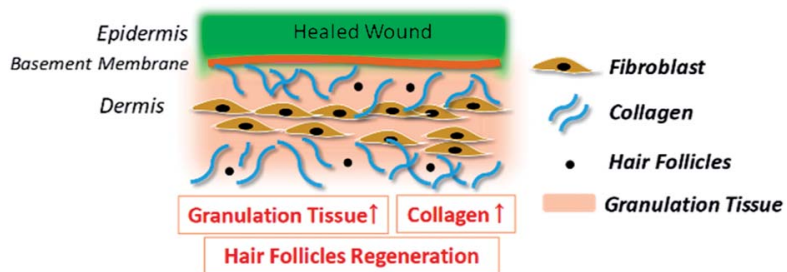

B

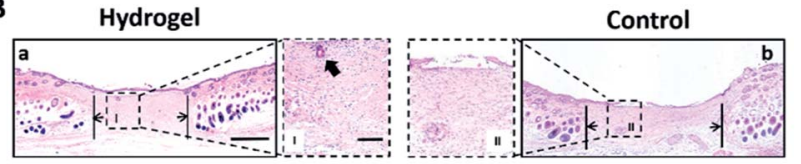

C

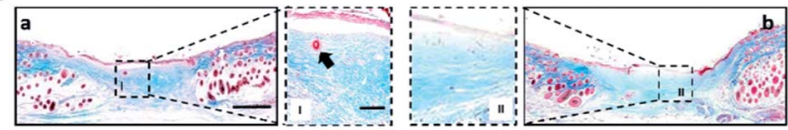

$D=4+* * \quad$ E
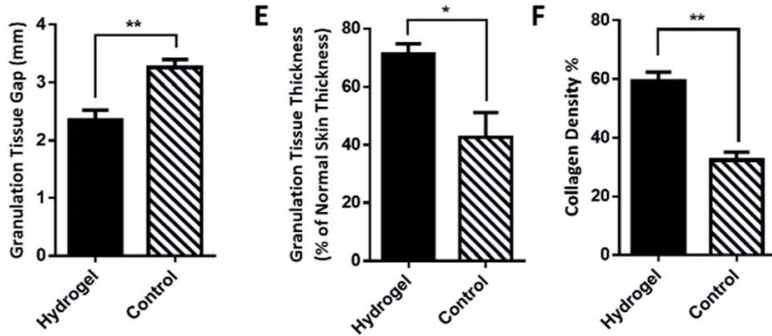

Fig. 3 Copolypeptide hydrogel enhances skin maturation and functionalization in a microscopic scale. (A) Schematic diagram of granulation tissue and collagen deposition. (B) H\&E staining on day 20 after treatment of hydrogel and saline (control). The granulation tissue areas I and II are magnified. The arrows in (a) and (b) indicated the granulation tissue gap. The black arrow in the magnified image I represented the newly regenerated hair follicles after hydrogel treatment. The scale bar in (a) and (b) is $1 \mathrm{~mm}$, and in magnified images I and II is $100 \mu \mathrm{m}$. (C) MT staining on day 20. The blue color indicates the distribution of the collagen. Area I and II in (a) and (b) are magnified. The black arrow in the magnified image represents new hair follicles generated after hydrogel treatment. The scale bar in (a) and (b) is $1 \mathrm{~mm}$, and in magnified images I and II is $100 \mu \mathrm{m}$. (D) Quantified granulation tissue gap on day 20. (E) Percentage of granulation tissue thickness of normal skin thickness on day 20. (F) Quantified collagen density on day 20 Statistical differences were performed using one-way analysis of variance (ANOVA). $* * P<0.01, * P<0.05$, compared to the control group, $n>3$.

distributed blue color compared with that of the control. Statistical calculation revealed that the blue color density was $59.33 \pm 2.96 \%$ for the hydrogel treated wound, which was remarkably higher than that of $32.33 \pm 2.73 \%$ of the control (Fig. 3F). These data proved that the hydrogel could effectively enhance the deposition of dense and homogenous collagen tissue in the wound site, which would regenerate a more normal skin. Meaningfully, new hair follicle in the wound site was observed in the hydrogel treated wound, which was indicated by black arrows in magnified images in both H\&E and MT staining figures (Fig. 3B and C). As one of the skin accessories, hair follicles assist the normal function of skin such as serving as cell reservoir to several kinds of stem cells and expediting skin healing after injury. ${ }^{19}$ Thus, the hair follicles restoration implied the high potent of the hydrogel on the normalization of the injured skin. To sum up, all the data above proved that the hydrogel could improve wound regeneration in a microscopic scale in vivo.

\subsection{The hydrogel enhanced angiogenesis}

As the hydrogel has been proved to accelerate wound healing macroscopically and microscopically, its mechanism on active wound regeneration was further investigated. Since in the inflammation stage of wound healing, the degradative enzymes release such as matrix metalloproteinase (MMPs) and serine proteases are up-regulated, ${ }^{20,21}$ we hypothesized that the hydrogel would be degraded to release glutamic acid and lysine, which was believed to enhance the angiogenesis and increase collagen deposition. ${ }^{9,22,23}$ To verify our hypothesis, the wounded skin samples of the early stage (day 7) were collected and their expression of cluster of differentiation 31 protein (CD-31) and $\alpha$ smooth muscle actin ( $\alpha$-SMA) were detected by immunofluorescence to study the regeneration degree of blood vessels. As shown in Fig. 4A, the structure of a mature blood vessel includes
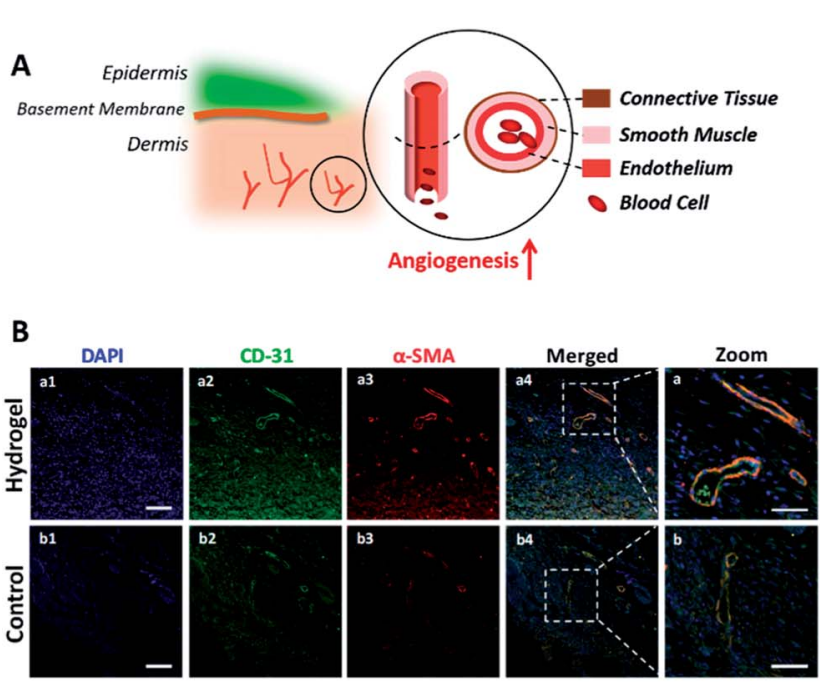

C

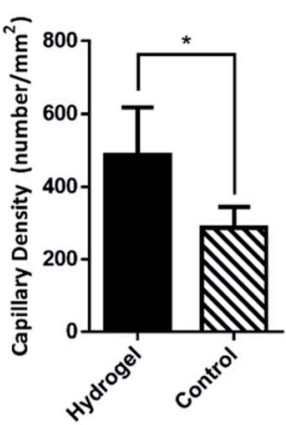

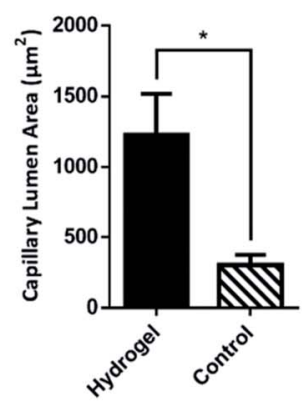

Fig. 4 Copolypeptide hydrogel facilitates vascular regeneration and maturation. (A) Illustration of the structure of blood vessels. (B) Immunofluorescent staining of CD-31 (green) and $\alpha$-SMA (red). The same scale bar of $100 \mu \mathrm{m}$ is used in (a1)-(a4) and (b1)-(b4), and $50 \mu \mathrm{m}$ in magnified images of (a) and (b). Blue color indicates the nuclei. The yellow color represents the co-localization of green and red colors. The selected area in (a4) and (b4) were magnified for further observation. (C) Quantified capillary density on day 7 of the two groups. (D) Quantified capillary lumen area on day 7 of the two groups. Statistical differences were performed using one-way analysis of variance (ANOVA). ${ }^{*} * P<0.05$, compared to the control group, $n>3$. 
endothelium and smooth muscles, thus the co-detection of the endothelial cell marker CD-31 and a smooth muscle cell marker $\alpha$-SMA would be more convincing to locate the newly formed blood vessels. Fig. 4B exhibited that the hydrogel could remarkably increase endothelial cells and smooth muscle cells in the wounds, which was shown as higher density of green and red fluorescence, in contrast with the control group, indicating the formation of blood vessels. The co-localization of green and red fluorescence (as show yellow in Fig. 4B-a) additionally convinced the extensively existence of blood vessels in the hydrogel treated wounds. Further statistical analysis in Fig. 4C showed that the hydrogel treated wounds had a blood vessels density of $488.50 \pm 64.37 \mathrm{~mm}^{-2}$, which was much higher than $286.80 \pm 25.94 \mathrm{~mm}^{-2}$ of the control group. Besides, based on fluorescence images in Fig. 4B, the lumen areas of capillaries in the wound site were also measured and the result summarized in Fig. 4D showed that the blood vessels in the hydrogel treated wounds has an average lumen area of $1128.32 \pm 291.03 \mu^{2}$, which was significantly larger than $304.38 \pm 71.95 \mu \mathrm{m}^{2}$ of the control, demonstrating that the hydrogel treatment could result in more mature and larger blood vessels in wounds. Since the blood vessels provide oxygen and nutrients to the wound site in support of cell growth and tissue regeneration, ${ }^{\mathbf{2 4 , 2 5}}$ the mature and large blood vessel formation in the hydrogel treated wounds could excessively benefit the wound regeneration by accommodating the high oxygen and nutrients consumption during the wound healing process.

\subsection{The hydrogel enhances re-epithelialization}

For rapid wound healing, the fibroblast, keratinocytes and other key cells should effectively proliferate to form granulation tissue, refill the wound gap and achieve re-epithelialization for wound closure (Fig. 5A). With sufficient oxygen and nutrients supplement, we believe that the wound treated with the hydrogel could more efficiently promote cell proliferation and epithelialization to accelerate wound healing. To validate our hypothesis, wounded skin samples were collected on day 7 and investigated by immunofluorescent staining. We firstly studied the effect of the hydrogel on the cell proliferation by detecting the expression of proliferating cell nuclear antigen (PCNA) in the wounded tissue. PCNA is a marker of cell proliferation which is specifically expressed in the cell nucleus during $S$ phase of the cell cycle. ${ }^{26}$ As shown in Fig. 5B-a2 and b2, the PCNA positive cells (red) were more abundant in the hydrogel treated wound in comparison with the control group, indicating that the hydrogel induced a faster cell proliferation. For reepithelialization process, proliferated cells, especially the basal cells, the bottom layer of keratinocytes, would ascend, differentiate and undergo morphological change to offer sufficient keratinocytes to close the epidermal wound gap. Since the hydrogel could greatly motivate cell proliferation, it was believed that the hydrogel would improve re-epithelialization by providing the injured epidermis with plentiful keratinocytes. To prove the assumption, cytokeratin, the epithelial keratinocyte expressing protein was fluorescently stained and observed under microscope. As shown in Fig. 5B-a3 and b3, the upper
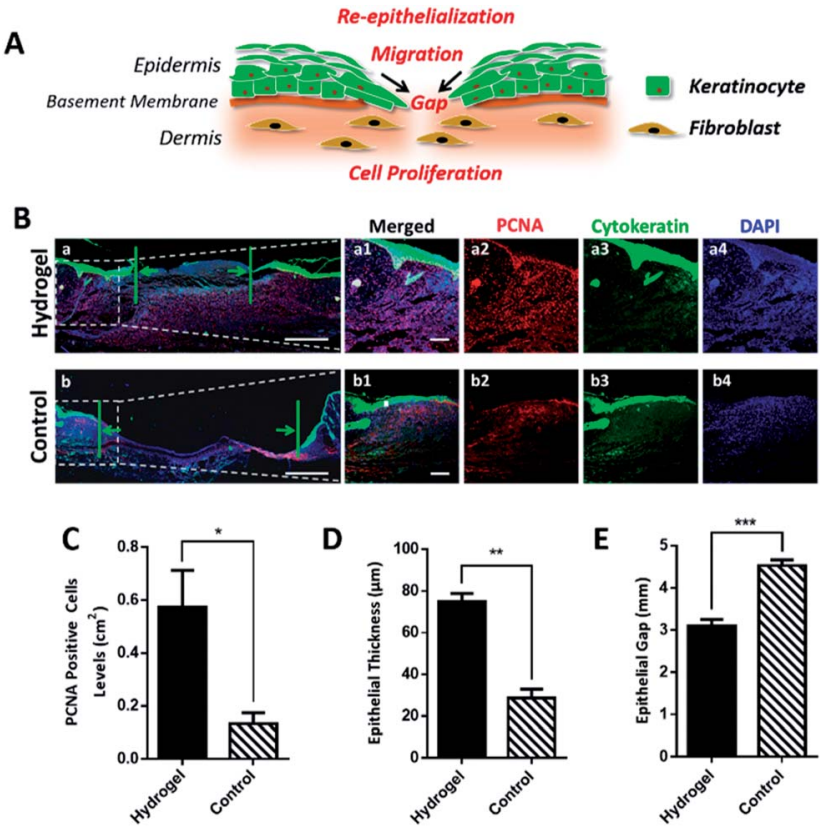

Fig. 5 Copolypeptide hydrogel promotes granulation tissue formation, keratinocyte cell proliferation and re-epithelialization. (A) Schematic diagram of the re-epithelialization process of wound healing, which is associated with cell proliferation and migration. Keratinocytes at the wound edge migrate towards the wound center to close the wound gap. (B) Immunofluorescent staining of cytokeratin (green) and proliferating cell nuclear antigen (PCNA, red). (a1)-(a4) and (b1)-(b4) are the minified images of selected areas in (a) and (b) with merged and split fluorescence channels. The scale bar in (a) and (b) is $1 \mathrm{~mm}$, and $100 \mu \mathrm{m}$ in magnified images. (C) Quantified PCNA positive cells levels on day 7 of the two groups. (D) Quantified epithelial thickness on day 7 of the two groups. (E) Quantified epithelial gap on day 7 of the two groups. Statistical differences were performed using one-way. analysis of variance (ANOVA). $* * * P$ value $<0.001, * * P<0.01, * P<0.05$, compared to the control group, $n>3$.

layer of cytokeratin-positive keratinocytes with green fluorescence in the hydrogel treated wound tissue was obviously thicker than that of the control group. Moreover, the epidermal gaps indicated by the green arrows in Fig. 5B-a and b demonstrated that the hydrogel quickened epithelialization of the wounds due to its narrower gap. All these data verified that the hydrogel was capable to enhance re-epithelialization of wounded skin. In consistent with the fluorescent images results, the statistic outcome (Fig. 5C-E) further confirmed that the hydrogel would promote cell proliferation and drive the wound re-epithelialization, which ultimately resulting in the effective wound regeneration both in macroscopic and microscopic scales.

\section{Conclusions}

In this study, the glutamic acid and lysine copolypeptide based biodegradable hybrid hydrogel was synthesized and applied to treat dermal wound. The hydrogel worked both as wound dressing and therapeutic prodrug to improve wound regeneration. In vivo result showed that the hydrogel could accelerate granulation tissue formation, increase collagen deposition and 
thus promote the wound closure. Further investigation proved that the hydrogel could facilitate vascular regeneration, which providing sufficient nutrients and oxygen to increase cell proliferation, migration and finally accelerate reepithelialization to achieve highly effective wound regeneration. All these data indicated that the peptide hydrogel could be a promising candidate for wound therapy.

\section{Conflicts of interest}

The authors declare no conflict of interest.

\section{Acknowledgements}

This work was financially supported by the National Natural Science Foundation of China [81701809, 8160161, 81571395, 81771531]. The authors also want to thank the financial support from Zhejiang Educational Committee of China (Y201737074), and Wenzhou Science \& Technology Bureau of China (Y20170157).

\section{Notes and references}

1 S. Guo and L. A. Dipietro, J. Dent. Res., 2010, 89, 219-229.

2 J. E. Janis, R. K. Kwon and D. H. Lalonde, Plast. Reconstr. Surg., 2010, 125, 230e-244e.

3 S. S. Anumolu, A. R. Menjoge, M. Deshmukh, D. Gerecke, S. Stein, J. Laskin and P. J. Sinko, Biomaterials, 2011, 32, 1204-1217.

4 M. Rafat, M. Xeroudaki, M. Koulikovska, P. Sherrell, F. Groth, P. Fagerholm and N. Lagali, Biomaterials, 2016, 83, 142-155.

5 S. Halstenberg, A. Panitch, S. Rizzi, H. Hall and J. A. Hubbell, Biomacromolecules, 2002, 3, 710-723.

6 O. Jeon, J. E. Samorezov and E. Alsberg, Acta Biomater., 2014, 10, 47-55.

7 G. Ma, W. Lin, Z. Yuan, J. Wu, H. Qian, L. Xu and S. Chen, J. Mater. Chem. B, 2017, 5, 935-943.

8 D. Oberleas, Nutr. Rev., 1967, 25, 125-127.

9 P. Thangavel, B. Ramachandran, S. Chakraborty, R. Kannan, S. Lonchin and V. Muthuvijayan, Sci. Rep., 2017, 7, 10701.

10 J. R. Hernandez and H. A. Klok, J. Polym. Sci., Part A: Polym. Chem., 2003, 41, 1167-1187.
11 H. Li and Y. Niu, Polym.-Plast. Technol. Eng., 2012, 51, 10621067.

12 Z. Wang, J. Wang, Y. Jin, Z. Luo, W. Yang, H. Xie, K. Huang and L. Wang, ACS Appl. Mater. Interfaces, 2015, 7, 2462924640.

13 R. Jayakumar, M. Prabaharan, P. T. Sudheesh Kumar, S. V. Nair and H. Tamura, Biotechnol. Adv., 2011, 29, 322-337.

14 C. I. Fiedler, E. A. Aisenbrey, J. A. Wahlquist, C. M. Heveran, V. L. Ferguson, S. J. Bryant and R. R. McLeod, Soft Matter, 2016, 12, 9095-9104.

15 A. H. Dewi, I. D. Ana and J. Jansen, J. Biomed. Mater. Res., Part A, 2017, 105, 1055-1062.

16 M. Takeo, W. Lee and M. Ito, Cold Spring Harbor Perspect. Med., 2015, 5, a023267.

17 L. Zhao, L. Niu, H. Liang, H. Tan, C. Liu and F. Zhu, ACS Appl. Mater. Interfaces, 2017, 9, 37563-37574.

18 N. Lohmann, L. Schirmer, P. Atallah, E. Wandel, R. A. Ferrer, C. Werner, J. C. Simon, S. Franz and U. Freudenberg, Sci. Transl. Med., 2017, 9, eaai9044.

19 C. M. Chuong, Nature, 2007, 447, 265-266.

20 G. Power, Z. Moore and T. O'Connor, J. Wound Care, 2017, 26, 381-397.

21 J. Douaiher, J. Succar, L. Lancerotto, M. F. Gurish, D. P. Orgill, M. J. Hamilton, S. A. Krilis and R. L. Stevens, Adv. Immunol., 2014, 122, 211-252.

22 X. Wang, B. Liu, Q. Xu, H. Sun, M. Shi, D. Wang, M. Guo, J. Yu, C. Zhao and B. Feng, Wound Repair Regen., 2017, 25, 270-278.

23 M. Thiersch, M. Rimann, V. Panagiotopoulou, E. Ozturk, T. Biedermann, M. Textor, T. C. Luhmann and H. Hall, Biomaterials, 2013, 34, 4173-4182.

24 F. Bossi, C. Tripodo, L. Rizzi, R. Bulla, C. Agostinis, C. Guarnotta, C. Munaut, G. Baldassarre, G. Papa, S. Zorzet, B. Ghebrehiwet, G. S. Ling, M. Botto and F. Tedesco, Proc. Natl. Acad. Sci. U. S. A., 2014, 111, 42094214.

25 C. L. Baum and C. J. Arpey, Dermatol. Surg., 2005, 31, 674686.

26 P. Kurki, M. Vanderlaan, F. Dolbeare, J. Gray and E. M. Tan, Exp. Cell Res., 1986, 166, 209-219. 